\title{
Identification of Genes Related to Arsenic Detoxification in Rice Roots Using Microarray Analysis
}

\author{
Quynh-Thi Thuy Nguyen, Tsai-Lien Huang, and Hao-Jen Huang
}

\begin{abstract}
Arsenic (As) contamination in natural environment is a problem in many countries. Plants exposed to As show cytotoxic effects as inhibit cellular function, disrupt metabolism and reduce the yield. However, little is known about the molecular mechanisms of rice early expose to arsenate $(\mathrm{AsV})$ at low concentration $(10 \mu \mathrm{M})$. Therefore, to better understand the molecular basis of rice cells, we performed a large-scale during analysis of the rice transcriptome within 24 hours challenged with AsV, one kind of common As inorganic species. In our microarray data, 614 transcripts were identified in response to AsV treatment. These transcripts related in plant defense mechanisms and the regulation of genes encoding to glutathione-S-transferase, oxidative stress, heat shock protein and enzymes in the ubiquitination pathway of protein degradation. Several important genes related to sulfate assimilation and GSH metabolism were induced for detoxification of As. Activities of peroxidase (POD) in rice roots increased after $12 \mathrm{~h}$ and $24 \mathrm{~h}$ treated to $\mathrm{AsV}$, while the glutathione content at $12 \mathrm{~h}$ displayed decreasing trend but increasing trend after $24 \mathrm{~h}$. Taken together, these data provide an overview of novel insights of AsV detoxification in rice and elicited for further investigation mechanism of arsenic detoxification in plants.
\end{abstract}

Index Terms-Arsenite, transcriptome, rice, glutathione.

\section{INTRODUCTION}

Arsenic (As) is a toxic metalloid present in ground and surface waters throughout the world [1]. Two kind of inorganic forms of As exits popularly in environment are arsenite (AsIII) and arsenate (AsV), which are more toxic than the organic forms. Both of inorganic forms are highly toxic and plants exposed to them show symptoms relating inhibited root growth, decreased photosynthetic rate and reduced in crop yield. As concentration in groundwater in many countries such as United State and Asians have reported excess $10 \mu \mathrm{g} / \mathrm{l}$, the permissible limit by WHO [2]. Arsenic in the groundwater can be used for irrigation that enters to the food-chain through plant up take and consumption by animals and humans. As concentration in rice and some vegetables are exceed the safety limits [3]. Previous studies have reported the molecular mechanism in plants during As stress [4], [5]. However, these studies carried out in the plants exposed to high As concentrations, little is known on rice roots subjected to low concentration of AsV. Moreover, rice (Oryza sativa L.) that is one of the

Manuscript received July 29, 2013; revised September 24, 2013. Quynh-Thi Thuy Nguyen and Tsai-Lien Huang contributed equally to this article.

Quynh-Thi Thuy Nguyen and Hao-Jen Huang are with Department of Life Sciences, National Cheng Kung University, Taiwan (e-mail: baokhanh0808@yahoo.com.vn).

Tsai-Lien Huang is with Center for General Eduction, Tzu Chi College of Technology, Taiwan. most popular cereals worldwide. Hence, we focused rice to study the molecular mechanism and identify gene expression during the early stages with AsV.

Under environmental stresses including the heavy metals disrupt the redox homeostasis of cells and produce rapidly of reactive oxygen species (ROS). At low concentration, ROS seem as a signal for repairing of cellular damage, but high levels may lead lipid peroxidation, enzyme inactivation and DNA and membrane damage. To defend against oxidative damage, plants produce enzymatic antioxidants such as peroxidase (POD) and non-enzymatic antioxidant such as glutathione (GSH) which play an important role in regulating the concentrations of cellular ROS [6].

Recently, plants have been known to detoxify and accumulate As in the root tissues. After entering into roots, $\mathrm{AsV}$ is reduced to AsIII which is subsequently detoxified by phytochelatins (PCs). Studies showed that phytochelatins (PCs) play an important role in the detoxification of As [4], or heavy metals [7]. AsV-PCs complexes sequestered into vacuoles by glutathione-conjugating pumps, and then form a more complex aggregation in the vacuole [8]. Two genes that belong to ATP binding cassette (ABC) transporter, AtABCC1 and AtABCC2, are major vacuolar PC transporters required for As detoxification [9].

Currently, cDNA microarray assay is a useful tool for understanding the plant regulatory mechanisms under biotic/abiotic stresses. Hence, the aims of our study were to understand molecular mechanism of As detoxification in rice roots response to AsV stress at low concentration utilizing a custom Agilent $44 \mathrm{~K}$ rice microarray. Our data indicated that the involvement of the stress-related genes and the genes related to anti-oxidation systems were positive regulated, and biosynthesis pathways were regulated. These findings expand that of previous studies about transcriptional responses and provide more clues to understanding the molecular and detoxification mechanism in rice under arsenic stress.

\section{MATERIAL AND MethodS}

\section{A. Plant Material, Growth and Stress Conditions}

Rice seeds (Oryza sativa L. cv. TN-67) were germinated in distilled water for 3 days at $26^{\circ} \mathrm{C}$ in darkness. Once the roots reached $3-4 \mathrm{~cm}$ in length, they were treated with different concentrations of $\mathrm{AsV}\left(\mathrm{Na}_{2} \mathrm{HAsO}_{4} .7 \mathrm{H}_{2} \mathrm{O}\right.$; Sigma, USA) during $3 \mathrm{~d}$, then root lengths were measured by metric scale. Experiments were repeated at least three times.

\section{B. RNA Purification, cDNA Synthesis and Semi- Quantitative RT-PCR}

Total RNA from rice roots, treated with or not AsV for 24 
h, was extracted using of the RNAeasy Plant Mini Kit (QIAGEN, Hilden, Germany). cDNA was performed from $1 \mu \mathrm{g}$ of total RNA from each sample with a ImProm-II Reverse Transcription System (Promega, USA). The primer sequences of selected genes for semi-quantitative RT-PCR were designed to span an exon-exon junction, eliminating amplification of genomic DNA. Tubulin gene was used as an internal control to ensure that equal amounts of cDNA in all the reactions. All experiments were repeated at least 3 times for each gene.

\section{Microarray Experiment and Transcriptome Analyses}

Total RNA of the rice roots were collected in control conditions and AsV-treated, which used as probes for the Agilent Rice Oligo microarray (44K Agilent Technologies, USA). Briefly $0.7 \mu \mathrm{g}$ of total RNA was amplified by a Fluorescent Linear Amplification Kit (Agilent Technologies, USA) and labeled with Cy3-CTP (control samples) or Cy5CTP (AsV-treated) (CyDye, PerkinElmer, USA). Hybridization at $60^{\circ} \mathrm{C}$ for $17 \mathrm{~h}$ in a rotating hybridization oven according to the manufacturer's protocol. Raw data were normalized by the GenSpringGX11 software (Agilent Technologies). Data were $\log 2$ converted and statistically analyzed by using Rank Products technique to identify differentially expressed genes with $P$ value $<0.001$ in the ttest. The Benjamini-Hochberg False Discovery Rate (FDR) method was calculated to obtain corrected $P$ values for multiple testing. Genes were considered as significantly up/down regulated if the FDR value for the corresponding probe set was $<0.15$. AsV-induced genes were classified into functional categories based on EasyGO functional enrichment analysis [10] and Mapman [11].

\section{Determine POD Activity}

For extraction of POD enzyme, AsV-treated roots were homogenized with $0.1 \mathrm{M}$ sodium phosphate buffer ( $\mathrm{pH}$ 6.8). The homogenate was centrifuged at $13,000 \mathrm{~g}$ for $15 \mathrm{~min}$ and the collecting supernatant was used for determination of enzyme activity. The whole extraction procedure was performed at $4^{\circ} \mathrm{C}$. POD was assayed as described previously [12].

\section{E. Measurement of Glutathione Content}

Total glutathione content was analyzed according to Anderson et al. (1995) [13]. About $0.1 \mathrm{~g}$ fresh rice roots were homogenized in $5 \%$ sulpho-salicylic acid. $100 \mu \mathrm{l}$ supernatant was collected and mixed to $700 \mu \mathrm{l}$ of $0.3 \mathrm{mM}$ of NADPH, $100 \mu \mathrm{l}$ of DTNB solution and $50 \mu \mathrm{l}$ of glutathione reductase $\left(10\right.$ units $\left.\mathrm{ml}^{-1}\right)$. The reaction mixture was measured at $412 \mathrm{~nm}$ at room temperature. GSH content was calculated by subtracting GSSG from the total glutathione (GSH+ GSSG).

\section{RESUlTS AND DISCUSSION}

\section{A. Effect of AsV Stress on Root Growth}

Arsenate (AsV) is considered to be among the most toxic to plants. To examine the its toxicity on rice seedlings, we performed a set period of time with different concentration of $\mathrm{AsV}$ solutions. The growth of rice roots exposed to 25 $\mu \mathrm{M}$ was completely inhibited, and the root elongation was reduced by approximately one-half at $10 \mu \mathrm{M}$ (Fig. 1). Therefore, the microarray assay was designed to identify genes that are expressed differently in rice roots treated with $10 \mu \mathrm{M}$ AsV for $24 \mathrm{~h}$.

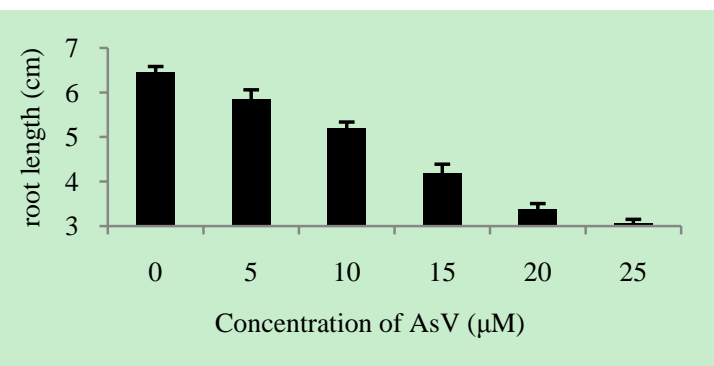

Fig. 1. Effect of different concentrations of AsV on rice root length.

\section{B. Identify AsV-Induced Genes Expression in Rice Roots}

To analysis the molecular mechanisms response to AsV, a $44 \mathrm{~K}$ cDNA microarray was applied to analyze the transcription levels of the rice roots exposed to the AsV $(10 \mu \mathrm{M}) .427$ up- and 187 down-regulated genes were found. Using the gene ontology (GO) annotation with an fdr adjusted p-value of $\leq 0.05$ as the cutoff [14], we identified the $8 \mathrm{GO}$ categories into which up-regulated transcripts (Table I). Most of the these genes were assigned into biological process and molecular function such as secondary metabolic process, response to stress, jasmonic acid (JA) biosynthetic process, glutathione transferase activity, chitin binding, oxygen binding and UDP-glycosyltransferase activity. Whereas, number of the down-regulated genes belonged the sulfotransferase activity, lipid metabolic process and endomembrane system terms were induced (Table I). It is interesting to note that 20 genes encoding to lipid metabolic process were down-regulated in rice roots exposed to AsV. Lipid metabolic process known that related to construction of cellular membrane system and biosynthesis endogenous necessary to cell expansion. Therefore, AsV may have a significant effect on the inhibition of root elongation by regulation cell membrane structure.

Numerous detoxification-related genes were up-/downregulated in rice roots subjected to $\mathrm{AsV}$. One gene encoding to Metallothionein (MTs, Os12g0568200) were found in our library. All of 14 genes encoding Cytochrome P450 (CYP) were observed to be upregulated. Using the Mapman software, we found also 10 putative regulatory genes encoding proteasome-related pathway under AsV treatment (Fig. 2a). These genes belong to various types of proteasome-related components such as RING/U-box superfamily protein (Os06g0159900), RING-H2 finger protein ATL40 (Os06g0537300) and RING-Zn finger proteins (Os01g0213400, Os02g0559800, Os05g0360400, Os07g0631200 and Os09g0243200). A total of 9 heat shock proteins (HSPs) were found to be up-regulated in rice root treated to AsV. Metallothioneins (MTs) have been known involved in maintaining the homeostasis of essential metals and function in non-essential metal detoxification [8]. Studies demonstrated that common pathway for the detoxification in most of organisms mediated by groups of enzymes as CYP. Chakrabarty et al. (2009) [15] indicated that the differently regulation of CYP from 10-days-old seedlings under higher concentration of $\mathrm{AsV}(25 \mu \mathrm{M})$ and 
AsV $(250 \mu \mathrm{M})$. Interestingly, at high concentration $(100 \mu \mathrm{M})$, AsV pointed out that has a dual effect on the Ub-dependent proteolytic pathway and caused its inhibition [16], but at lower concentration the Ub-conjugation enzymes are increase [17]. Hence, we suggest that the CYPs and $\mathrm{Ub} /$ protease-related proteins are necessary to counteract the toxicity induced by $\mathrm{AsV}$ in rice.

TABLE I: GENE ONTOLOGY ANALYSIS OF UP- AND DOWN-REGULATED BY ASV STRESS

\begin{tabular}{|c|c|c|c|c|}
\hline GO term & GO name & B/Rnum & Qnum & FDR pv \\
\hline \multicolumn{5}{|l|}{ Upregulated } \\
\hline \multicolumn{5}{|l|}{ Biological Process } \\
\hline GO:0009861 & jasmonic acid synthesis & 63 & 7 & 0.00818 \\
\hline GO:0006950 & response to stress & 1462 & 52 & $1.76 \mathrm{E}-05$ \\
\hline GO:0009266 & response to temperature stimulus & 255 & 23 & $2.20 \mathrm{E}-08$ \\
\hline GO:0044248 & cellular catabolic process & 837 & 33 & 0.000488 \\
\hline \multicolumn{5}{|l|}{ MolecularFunction } \\
\hline GO:0004364 & glutathione transferase activity & 113 & 28 & $3.55 \mathrm{E}-20$ \\
\hline GO:0019825 & oxygen binding & 305 & 11 & 0.000276 \\
\hline GO:0008194 & UDP-glycosyltransferase activity & 515 & 14 & 0.00567 \\
\hline GO:0003824 & catalytic activity & 11789 & 225 & 0.0438 \\
\hline \multicolumn{5}{|l|}{ Downregulated } \\
\hline \multicolumn{5}{|l|}{ Biological Process } \\
\hline GO:0006629 & lipid metabolic process & 1185 & 20 & 0.00362 \\
\hline \multicolumn{5}{|l|}{ Molecular Function } \\
\hline GO:0008146 & sulfotransferase activity & 88 & 8 & 0.000193 \\
\hline \multicolumn{5}{|l|}{ Cellular Component } \\
\hline GO:0012505 & endomembrane system & 3588 & 53 & $3.41 \mathrm{E}-05$ \\
\hline
\end{tabular}

Qnum: Number of query list; B/Rnum: Number of Background

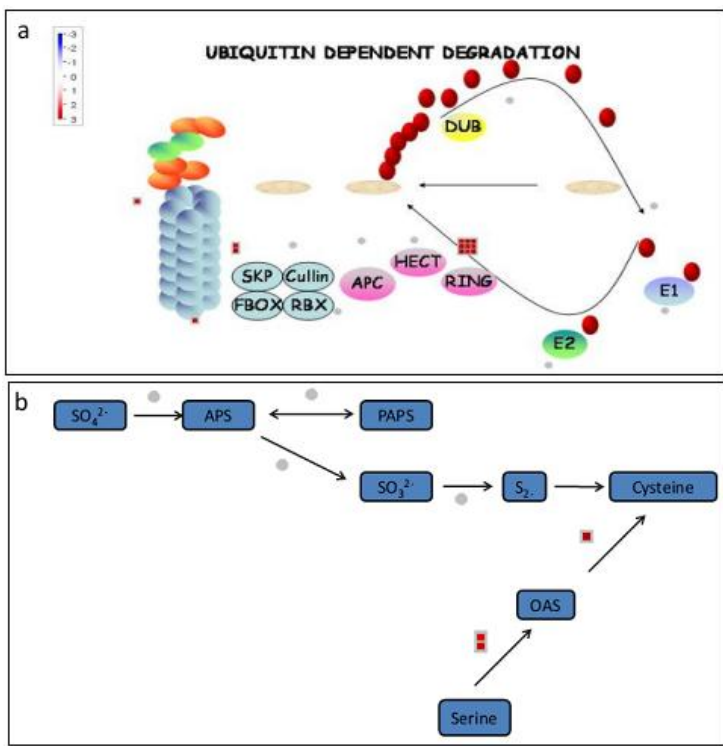

Fig. 2. Mapman analysis of genes related to a proteasome-related pathways and $b$ sulfate assimilation and Each BIN or subBIN is represented as a block, with each transcript revealed as square, red for up-regulation or blue for down-regulation.

Our microarray data revealed that various reactive oxidative stress (ROS)-related genes were to be up-/downregulated. 7 genes encoding to peroxidase were found to be up- and down-regulated in rice roots subjected to AsV. It suggested that AsV activated specific responses to the plant antioxidant defense system [18]. To confirm the observation above, we determined the enzymatic activity of POD in the rice roots with $\mathrm{AsV}$ at two time points (12h and $24 \mathrm{~h}$ treatments) (Fig. 3a). The result showed that the activity of POD increased significantly in response to $\mathrm{AsV}$ in both of time points, suggest that in rice plants POD may play important role in detoxification of $\mathrm{H}_{2} \mathrm{O}_{2}$ generated in the cellular.
Among genes reactive oxidative stress (ROS)-related, we found two genes encoding to Glutaredoxin (Grx: Os01g0241400 and Os02g0618100) were up-regulated. Especially, numerous of gene encoding to Glutathione Stransferase (GST) was significantly enrichment among the reactive oxygen species-related gene families. Studies showed that reactive oxygen species (ROS)-associated genes induced by heavy metals [19]. Glutaredoxin that is thought to mediate to glutathione biochemistry also showed up-regulated expression in our study. Both of two Grx genes have been reported in increased more than fivefold in rice roots subjected to juglone [9]. The up-regulated of GSTs has been shown in response to various heavy metals [20]. GSTs are known to catalyze the conjugation of heavy metals with reduced glutathione (GSH) and target them for ATPdependent transport into the vacuole. Especially, three genes related to sulfate assimilation were up-regulated significantly by the AsV treatment (Fig. 2b). Among them, two cysteine biosynthesis-related genes encoding serine acetyltransferse (SAT, Os03g19660 and Os03g018500) and one Multidrug-resistance associated protein (Os06g0158900). GSTs are known to catalyze the conjugation of toxic molecules such as heavy metals with reduced glutathione $(\mathrm{GSH})$ and target them for ATPdependent transport into the vacuole. The sulfate assimilation plays an important role in synthesizing cysteine, a precursor of glutathione biosynthesis [21]. Moreover, the activity of SAT has also shown increase in response to arsenic in plants [22], [23]. Together with our findings and the previous studies, we suggest that activity of SAT controls GSH biosynthesis in AsV detoxification in rice.

Our results showed that GSH content immediately decreased during $12 \mathrm{~h}$ treatment of $\mathrm{AsV}$, then gradually increase during 24 h (Fig. 3b). Depletion of GSH observed 
in Arabidopsis plants subjected to arsenate [24]. GSH levels would be rapidly depleted due to phytochelatins (PCs) production that leading to change in the equilibrium of ROS concentration and antioxidant enzymes in the cells [25].

A large number of transporter-related genes were found belonging to ATP-dependent transporters, secondary transporter and ion channels are expressed in AsV treatment Among them, $\mathrm{ABC}$ transporters are known in the transport of various compounds across biology membranes as well as phospholipids, ions, xenobiotics and heavy metals in plants and animals [26]. 11 genes encoding to $\mathrm{ABC}$ transporters were found to be up-regulated more than 7-fold in our study (Table 2). Studies have been shown that ABC transporters are concerned in arsenic detoxification by transporting GSH-complexed As [27], [28]. The up-regulation of two ABC transporters transcriptions (Os01g0695800, Os04g0209200) were analyzed in our study, as well as in other report under Cd stress in rice roots [29].

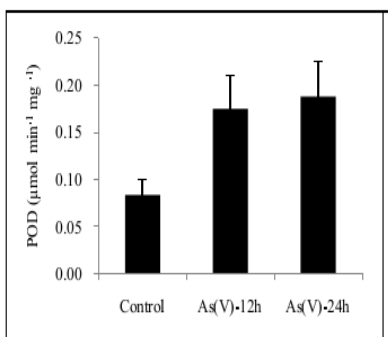

(a)

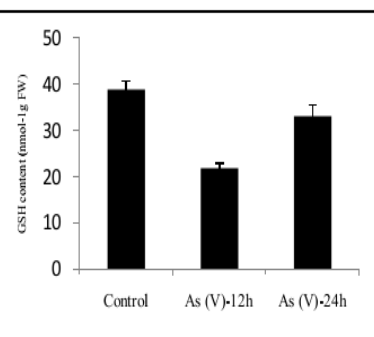

(b)
Fig. 3. Activity of (a) Peroxidase (POD) activity and (b) glutathione (GSH) content in rice roots subjected to $\mathrm{AsV}$.

TABLE II: TRANSCRIPTS ENCODING TO ABC TRANSPORTERS RESPONSIVE

\begin{tabular}{|c|c|c|}
\hline locus ID & Fdr & FC \\
\hline Os01g0615500 & 0.1180 & 7.19 \\
\hline Os01g0695800 & 0.0927 & 8.42 \\
\hline Os01g0836600 & 0.0168 & 22.39 \\
\hline Os01g0695800 & 0.0927 & 8.42 \\
\hline Os01g0836600 & 0.0168 & 22.39 \\
\hline Os03g0281900 & 0.1014 & 7.90 \\
\hline Os04g0209200 & 0.1039 & 7.61 \\
\hline Os05g0120000 & 0.0640 & 10.53 \\
\hline Os05g0120200 & 0.0496 & 12.44 \\
\hline Os05g0384600 & 0.0641 & 10.54 \\
\hline Os06g0158900 & 0.1172 & 7.37 \\
\hline
\end{tabular}

Fdr: false discovery rate; FC: $\log 2$ fold change

Transcription factors (TFs) and signaling regulators are known to be the most important category of genes related to expression of downstream genes that induced by abiotic stress [30]. 16 TFs genes which were identified in our microarray analysis. The expression of three genes encoding to MYB (Os10g0562100, Os01g0685400 and Os04g0593200) were increased. The MYB family members play important roles in response to biotic and abitotic stresses in plants [31]. Borevitz (2000) [32] reported that several MYP transcription factors that regulated the transcription of number genes related to Glutathione-Stransferase. One basic helix-loop-helix (bHLH) transcription factors genes (Os10g0556200) were up-regulated in AsV stress. The overexpression of OsbHLH148 in transgenic plants related to tolerance to drought stress [33]. Especially, bZIP10 (Os01g0859300) was up-regulated significantly forty-fold from rice roots subjected to $\mathrm{AsV}$. The expression of OsbZIP10 reported that induced by drought, salt and cold stress treatments [34]. Thus, we could suggest that these transcription factors were regulatory factors in rice seedlings subjected with AsV low concentrations at early stage.

In our study, most all of the AsV-responsive kinases were associated with tyrosine kinase-like (TKL) groups and were to be up-/down-regulated. Recent studies revealed that role of TKL involved in abiotic stress responses [35], [36]. Base on these reports, we assumed that protein kinases may be involved in defense and tolerance response under $\mathrm{AsV}$ treatment condition.

In order to validate gene expression changes determined by microarray, RT-PCR was conducted on five selected genes related to different pathways. The RT-PCR results showed similar to microarray data obtained in the microarray analysis (Fig. 4).

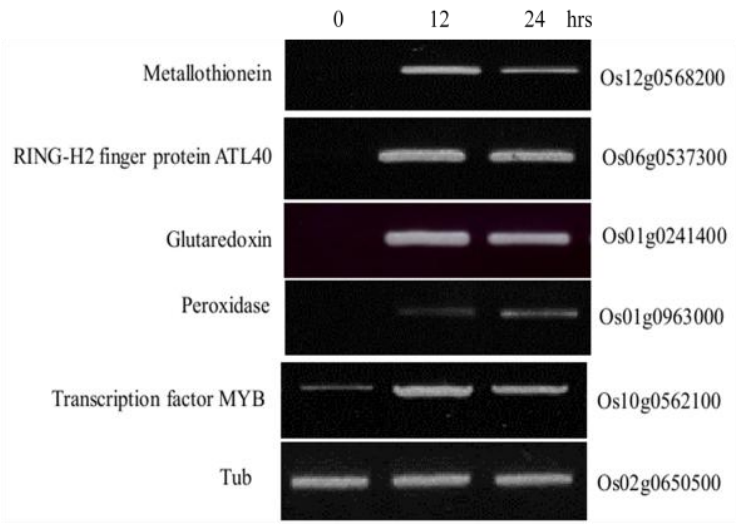

Fig. 4. Verification of microarray results by RT-PCR

In conclusion, our microarray results suggest that detoxification proteins such as MT, CYP and ROS scavengers are related to protection against AsV toxicity. In addition, more regulation function-related proteins including protein transporters, kianses and TFs play an important role in AsV stress in rice seedlings (Fig. 5). Taken together, our data provide insights that lead to a further understanding of molecular and physiological response to the early stage of AsV stress in rice plants.

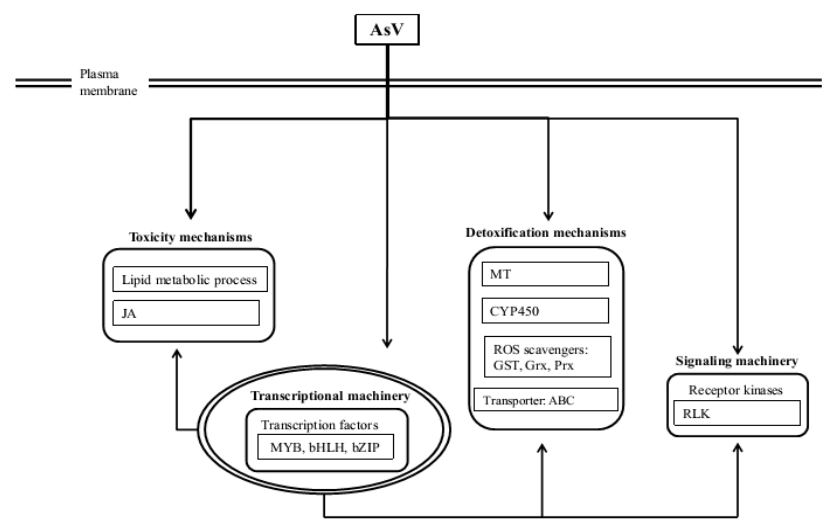

Fig. 5. Molecular mechanisms of AsV detoxification and protection in rice roots. 


\section{REFERENCES}

[1] J. O. Nriagu, "Arsenic poisoning through the ages," Environmental chemistry of arsenic, New York, NY, USA: Marcel Dekker, pp. 1-26, 2002.

[2] G. P. Bienert, M. Thorsen, M. D. Schüssler, H. R. Nilsson, A. Wagner, M. J. Tamás, and T. P. Jahn, "A subgroup of plant aquaporins facilitate the bi-directional diffusion of $\mathrm{As}(\mathrm{OH})_{3}$ and $\mathrm{Sb}(\mathrm{OH})_{3}$ a cross membranes," BMC Biology, vol. 6, pp. 26, June 2008

[3] H. K. Das, A. K. Mitrab, P. K. Senguptac, A. Hossaind, F. Islame, and G. H. Rabbanif, "Arsenic concentrations in rice, vegetables, and fish in Bangladesh: a preliminary study," Environment International, vol. 30, no. 3, pp. 383-387, May 2004.

[4] A. Raab, H. Schat, A. A. Meharg, and J. Feldmann, "Uptake, translocation and transformation of arsenate and arsenite in sunflower (Helianthus annuus): formation of arsenic-phytochelatin complexes during exposure to high arsenic concentrations," New Phytologist, vol. 168, no. 3, pp. 551-558, Dec. 2005.

[5] B. Paulose1, S. Kandasamy, and O. P. Dhankher, "Expression profiling of Crambe abyssinica under arsenate stress identifies genes and gene networks involved in arsenic metabolism and detoxification,' BMC Plant Biology, vol. 10, June 2010.

[6] S. K. Panda, R. K. Upadhyay, and S. Nath, "Arsenic stress in plants," Journal of Agronomy and Crop Science, vol. 196, no. 3, pp. 161-174, June 2010.

[7] Y. L. Zhu, E. A. H. Pilon-Smits, A. S.Tarun, S. U. Weber, L. Jouanin, and N. Terry, "Cadmium tolerance and accumulation in Indian mustard is enhanced by overexpressing-Glutamylcysteine synthetase," Plant Physiology, vol. 121, pp. 1169-1177, 1999.

[8] Cobbett and Goldsbrough, "Phytochelatins and metallothioneins: Roles in heavy metal detoxification and homeostasis," Аnnu. Rev. Plant Biol, vol. 53, pp.159-82, 2002

[9] W. Song et al., "Arsenic tolerance in Arabidopsis is mediated by two ABCC-type phytochelatin transporters," in Proc. Natl Acad. Sci. USA, vol. 107, no. 49, pp. 21187-21192, Sep. 2010.

[10] X. Zhou and Z. Su, "EasyGO: Gene Ontology-based annotation and functional enrichment analysis tool for agronomical species," $B M C$ Genomics, vol. 8, pp.246, July 2007.

[11] O. Thimm, O. Bläsing, Y. Gibon, A. Nagel, S. Meyer, P. Krüger, J. Selbig, L. A. Müller, S. Y. Rhee, and M. Stitt, "Mapman: a userdriven tool to display genomics data sets onto diagrams of metabolic pathways and other biological processes," The Plant Journal, vol. 37, no. 6, pp. 914-939, March 2004.

[12] C. J. Chang and C. J Kao, " $\mathrm{H}_{2} \mathrm{O}_{2}$ metabolism during senescence of rice leaves: changes in enzyme activities in light and darkness," Plant Growth Regulation, vol. 25, no. 1, pp.11-15, June 1998.

[13] M. D. Anderson, T. K. Prasad, and C. R. Stewart, "Changes in isozyme profiles of catalase, peroxidase, and glutathione reductase during acclimation to chilling in mesocotyls of maize seedlings," Plant Physiology, vol. 109, no. 4, pp. 1247-1257, Dec. 1995.

[14] T. Werner, "Bioinformatics applications for pathway analysis of microarray data," Current Opinion in Biotechnology, vol. 19, no. 1, pp. 50-54, Feb. 2008

[15] D. Chakrabarty et al., "Comparative transcriptome analysis of arsenate and arsenite stresses in rice seedlings," Chemosphere, vol. 74 no. 5, pp. 688-702, Feb. 2009.

[16] N. S. Klemperer and C. M. Pickart, "Arsenite inhibits two steps in the ubiquitin-dependent proteolytic pathway," J. Biol. Chem, vol. 264, no. 32, pp. 19245-19252, Nov. 1989.

[17] D. S. Kirkpatrick, K. V. Dale, J. M. Catania, and A. J Gandolf, "Lowlevel arsenite causes accumulation of ubiquitinated proteins in rabbit renal cortical slices and HEK293 cells," Toxicology and Applied Pharmacology, vol.186, no. 2, pp.101-109, Jan. 2003.

[18] A. Malecka, W. Jarmuszkiewiez, and B. Tomazewska, "Antioxidative defense to lead stress in subcellular compartments of pea root cells," Acta Biochim., vol. 40, pp. 687-698, Mar. 2001.

[19] D. Chandran, N. Sharopova, K. A. VandenBosch, D. F. Garvin, and D. A. Samac, "Physiological and molecular characterization of aluminum resistance in Medicago truncatula," BMC Plant Biology, vol. 8, no. 89, Aug. 2008.

[20] K. Lee et al., "Comparative proteomic analysis of the short-term responses of rice roots and leaves to cadmium," Journal of Plant Physiology, vol. 167, no. 3, pp. 161-168, Feb. 2010.

[21] S. Kopriva and H. Rennenberg, "Control of sulphate assimilation and glutathione synthesis: interaction with $\mathrm{N}$ and C metabolism," Journal of Experimental Botany, vol. 55, no. 404, pp.1831-1842, July 2004.

[22] S. Srivastava, A. K. Srivastava, P. Suprasanna, and S. F. SD'Souza, "Comparative biochemical and transcriptional profiling of two contrasting varieties of Brassica juncea L. in response to arsenic exposure reveals mechanisms of stress perception and tolerance,"
Journal of Experimental Botany, vol. 60, no. 12, pp. 3419-3431, June 2009.

[23] P. Tripathia, A. Mishraa, S. Dwivedia, D. Chakrabartya, P. K. Trivedia, R. B Singhb, and R. D. Tripathi, "Differential response of oxidative stress and thiol metabolism in contrasting rice genotypes for arsenic tolerance," Ecotoxicology and Environmental Safety, vol. 79, no. 1, pp.189-198, May 2012.

[24] Y. Li, O. P. Dhankher, L. Carreira, R. S. Balish, and R. B. Meagher, "Arsenic and mercury tolerance and cadmium sensitivity in Arabidopsis plants expressing bacterial-glutamylcysteine synthetase," Environmental Toxicology and Chemistry, vol. 24, no. 6, pp. 13761386, June 2005.

[25] G. Ainsworth et al., "Copper- and arsenate-induced oxidative stress in Holcus lanatus L. clones with differential sensitivity," Plant, Cell and Environment, vol. 24, no. 7, pp. 713-722, July 2001.

[26] K. Zientaza et al., "Activity of the AtMRP3 promoter in transgenic Arabidopsis thaliana and Nicotiana tabacum plants is increased by cadmium, nickel, arsenic, cobalt and lead but not by zinc and iron," Journal of Biotechnology, vol. 139, no. 3, pp. 258-263, Feb. 2009.

[27] E. M. Leslie, R. G. Deeley, and S. P. C Cole, "Toxicological relevance of the multidrug resistance protein 1, MRP1 (ABCC1) and related transporters," Toxicology, vol. 167, no. 1, pp. 3-23, Oct. 2001.

[28] P. M. Bleeker, H. W. J Hakvoort, M. Bliek, E. Soure, and H. Schat, "Enhanced arsenate reduction by a CDC25-like tyrosine phosphatase explains increased phytochelatin accumulation in arsenate-tolerant Holcus lanatus," The Plant Journal, vol. 45, no. 6, pp. 917-929, Mar. 2006.

[29] I. Ogawa, H. Nakanishi, S. Mori, and N. K. Nishizawa, "Time course analysis of gene regulation under cadmium stress in rice," Plant and Soil, vol. 325, no. 1-2, pp. 97-108, Dec. 2009.

[30] E. A. Bray, "Genes commonly regulated by water-deficit stress in Arabidopsis thaliana," Journal of Experimental botany, vol. 55, no. 407, pp. 2331-2341, Sep. 2004.

[31] M. R. Hemm, K. M. Herrmann, and C. Chapple, "AtMYB4: a transcription factor general in the battle against UV," Trends in Plant Science, vol. 6, no. 4, pp. 135-136, April 2001.

[32] J. O. Borevitz, Y. Xia, J. Blount, R. A. Dixon, and C. Lamb, "Activation tagging identifies a conserved MYB regulator of phenylpropanoid biosynthesis," The Plant Cell, vol. 12, no. 12, pp. 2383-2393, Dec. 2000.

[33] J. S. Seo et al., "OsbHLH148, a basic helix-loop helix protein, interacts with OsJAZ proteins in a jasmonate signaling pathway leading to drought tolerance in rice," The Plant Journal, vol. 65, no. 6, pp.907-921, Mar. 2011.

[34] G. Lu, C. Gao, X. Zheng, and B. Han, "Identification of OsbZIP72 as a positive regulator of ABA response and drought tolerance in rice," Planta, vol. 229, no. 3, pp.605-615, Feb. 2009.

[35] J. Giri, S. Vij, P. K. Dansana, and A. Tyagi, "Rice A20/AN1 zincfinger containing stress-associated proteins (SAP1/11) and a receptorlike cytoplasmic kinase (OsRLCK253) interact via A20 zinc-finger and confer abiotic stress tolerance in transgenic Arabidopsis plants," New Phytologist, vol. 191, no. 3, pp. 721-732, Aug. 2011.

[36] H. Tanaka et al., "Abiotic stress-inducible receptor-like kinases negatively control ABA signaling in Arabidopsis," The Plant Journal, vol. 70, no. 4, pp. 599-613, May 2012.

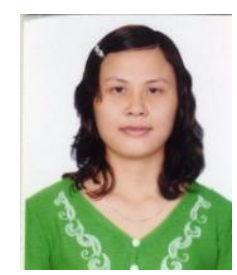

Quynh Thi Thuy Nguyen was born in Vietnam in 1978. She obtained her master's degree in biochemistry at Hanoi University of Science, VNU, Vietnam (2008). And she received her bachelor's in biochemistry at Hanoi University of Science, VNU, Vietnam (2000)

She is a PhD student in the Department of Life Sciences, National Chengkung University, Taiwan. Her research interest includes Bioactive compounds from medicinal plants, and Plant molecular of biology.

Mrs. Nguyen has a lot of publications (Study on chemical composition and hypoglycemic effect of some natural compounds from Tinospra sinensis on STZ mice induced -diabetic, J. Pharmacy, vol. 378, pp. 8-12 2007).

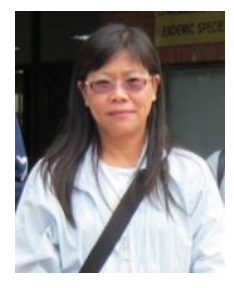

Tsai Lien Huang was born in Taiwan in 1962. She received her Doctor's degree in plant molecular of biology at National Chengkung University, Taiwan (2009), and master's degree in College of Medicine at National Taiwan University, Taiwan in 1994.

She is an assistant professor in Center for General Eduction, Tzu Chi College of Technology. Her research interest is plant molecular of biology.

Dr. Huang has a lot of publications (Early events 
in the signalling pathway for the activation of MAPKs in rice roots exposed to nickel, Functional Plant Biology, vol. 34, pp. 995-1001, 2007).

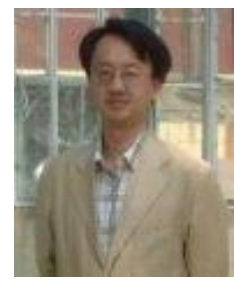

Hao-Jen Huang was born in Taiwan in 1967. He received his doctor's degree in biochemistry, University of Oxford, UK (1998), and master's degree in botany, National Taiwan University, Taiwan (1993), and bachelor's degree in Department of Biology, National Cheng Kung University, Taiwan (1989).

$\mathrm{He}$ is a professor in the Department of Life Sciences, National Chengkung University, Taiwan.
His research interest include plant molecular of biology, plant signaling.

Pro. Huang has a lot of publications (PaPTP1, a gene encoding protein tyrosine phosphatase from orchid, Phalaenopsis amabilis, is regulated during floral development and induced by wounding, Plant Molecular Biology Reporter, vol. 29, pp. 106-116, 2011). 